Journal of

Cancer Research and Therapeutic Oncology

\title{
Dabigatran and Cisplatin Co-Treatment Enhances the Antitumor Efficacy of Immune Checkpoint Blockade in A Murine Model of Resistant Ovarian Cancer
}

Eric T. Alexander ${ }^{1}$, Allyson R. Minton ${ }^{1}$, Molly C. Peters ${ }^{1}$, Joanne van Ryn ${ }^{2}$, Susan K. Gilmour ${ }^{{ }^{*}}$

${ }^{1}$ Lankenau Institute for Medical Research, Wynnewood, PA 19096 USA

${ }^{2}$ Boehringer Ingelheim Pharma GmbH \& Co. KG, Birkendofer Str. 65, 88397 Biberach an der Riss, Germany

*Corresponding author: Susan K. Gilmour, Lankenau Institute for Medical Research, 100 Lancaster Avenue, Wynnewood, PA 19096. Phone: 484-476-8429; Fax: 484-476-2205; Email: gilmours@mlhs.org

Received Date: January 06, 2019 Accepted Date: January 25, 2020 Published Date: January 28, 2020

Citation: Eric T. Alexander et al. (2020) Dabigatran and Cisplatin Co-Treatment Enhances the Antitumor Efficacy of Immune Checkpoint Blockade in A Murine Model of Resistant Ovarian Cancer. J Cancer Res Therap Oncol 8: 1-12.

\begin{abstract}
The standard treatment for ovarian cancer is surgical debulking followed by platinum- taxane-based chemotherapy. Although most patients are initially responsive to this therapy, patients in advanced stages eventually relapse and die. New therapeutic approaches using immune checkpoint blockade (ICB) have been less promising in ovarian cancer compared to other tumor types, resulting in durable tumor regression in only a small subset of ovarian cancer patients. Because previous studies showed immunomodulatory effects following co-treatment with cisplatin and the thrombin inhibitor dabigatran etexilate $(\mathrm{C} / \mathrm{D})$ in a preclinical animal model of ovarian cancer, we explored to what extent this co-treatment may enhance the anti-tumor efficacy of ICB in the ID8 tumor model that is resistant to ICB. Whereas cisplatin or dabigatran treatment alone or co-treatment with cisplatin and anti-PD-1 monoclonal antibody (mAb) demonstrated little significant effect on tumor spread, co-treatment with C/D with or without anti-PD-1 mAb significantly reduced ID8 tumor burden and increased peritoneal INF- $\gamma$ producing $\mathrm{CD} 8^{+}$T-cells after only 2 weeks of treatment. Moreover, $\mathrm{C} / \mathrm{D}$ cotreatment with ICB conferred a durable survival advantage over $\mathrm{C} / \mathrm{D}$ or ICB alone.
\end{abstract}

The enhanced anti-tumor effect and survival with $\mathrm{C} / \mathrm{D}$ co-treatment and ICB compared to that with $\mathrm{C} / \mathrm{D}$ or ICB alone was accompanied by decreases in immunosuppressive M2- macrophages, decreases in pro-tumorigenic cytokines, and corresponding increases in tumor-infiltrating, IFN- $\gamma$-producing CD ${ }^{+}$T-cells. Our findings provide proof-of-concept evidence that the addition of ICB with thrombin inhibition in frontline platinum-based chemotherapy may be a potential new therapeutic treatment combination for advanced ovarian cancer.

Keywords: Dabigatran; Thrombin; Ovarian Cancer; PD-1; CTLA-4

(C)2020 The Authors. Published by the JScholar under the terms of the Creative Commons Attribution License http://creativecommons.org/licenses/ by/3.0/, which permits unrestricted use, provided the original author and source are credited. 


\section{Introduction}

Although ovarian cancer accounts for only 3\% of all cancer in women, it is the fifth leading cause of cancer-related deaths for women in the United States [1]. Patients typically present with advanced-stage disease, and they often initially respond well to standard primary treatment with surgery and firstline platinum and taxane-based chemotherapy. However, the majority of patients experience recurrence of their cancer within 12-18 months and die of the disease [2]. Clearly, there is an urgent need for new therapeutic strategies for treating ovarian cancer. An exciting new cancer immunotherapy approach is to block two key immune checkpoint pathways mediated by immunosuppressive co-signaling, the first via programmed cell death-1 (PD-1) and programmed death ligand-1 (PD-L1) and the second via CTL-associated antigen 4 (CTLA-4) and its ligands B7-1 or B7-2 [3,4]. The immune checkpoint proteins, CTLA-4 and PD-1, normally keep immune responses in check by preventing overly intense responses that might damage normal tissue. Tumors can hijack these immune checkpoint proteins and use them to suppress immune responses. Blocking the activity of immune checkpoint proteins releases the "brakes" on the immune system, thus increasing its ability to destroy tumor cells. These new ICI treatments have led to dramatic tumor regressions in patients with some solid malignancies, including ovarian cancer $[5,6]$. Unfortunately, clinical studies have shown that the administration of inhibitors of CTLA-4, PD-1, and PD-L1 alone leads to durable tumor regression in only a subset of cancer patients [7, 8]. In ovarian cancer patients treated with immune checkpoint blockade, symptomatic disease progression is common and often leads to early discontinuation of treatment [9]. Because tumors employ multiple and non-overlapping immunosuppressive mechanisms that can mitigate the clinical benefit of immunotherapy such as immune checkpoint blockers, it is important to identify and block these resistance mechanisms.

The clinical association between cancer and thrombosis has been recognized for more than a century [10], and expression of coagulation factors and biomarkers of hemostatic system activation correlates strongly with poor prognosis for multiple cancer types [11-13]. Indeed, ovarian cancer is associated with a high risk of thrombotic events (20\%) which sometimes can be exacerbated by treatment with standard chemotherapeutic agents [14-16]. The pro-thrombotic microenvironment in tumors also directly promotes tumor growth and metastasis [17]. Thrombin is the primary effector protease of the coagulation cascade generated by the action of tissue factor and other coagu- lation factors. The critical role of thrombin in promoting tumor progression reflects its many functions, including fibrin formation [18], platelet activation [19], activation of protease-activated receptor (PAR) signaling [20] and the proteolytic breakdown of the extracellular matrix. In addition to its role in generating fibrin to promote hemostasis, thrombin acts directly on multiple effector cells of the immune system affecting both acute and chronic inflammatory processes $[21,22]$. The ablation of PAR-1 from the tumor microenvironment, but not the tumor, has been shown to dramatically reduce tumor growth and metastasis in multiple tumor models $[23,24]$, in part by reducing infiltration of M2-like macrophages into the tumor [23]. Thrombin-activated platelets release immunosuppressive cytokines including TGF- $\beta$ that can inhibit natural killer cell activity, helping the tumor evade host immunosurveillance $[25,26]$. Taken together, there is strong evidence that thrombin influences cancer pathogenesis via multiple mechanisms, including the tumor immune response, with thrombin emerging as a target for novel therapies in cancer. Using the murine ID8 ovarian tumor model, we have shown that the thrombin inhibitor, dabigatran etexilate, significantly enhances the anti-tumor efficacy of cisplatin in an immunomodulatory way [27]. Dabigatran is an oral anticoagulant that is a direct thrombin inhibitor [28]. The anti-tumor effect of this co-treatment was significantly greater than the reduction in tumor load from either cisplatin or dabigatran alone. The present investigation was designed to explore to what extent cisplatin and dabigatran co-treatment, that decreases the tumor infiltration of myeloid immunosuppressive cells, may enhance the efficacy of immune checkpoint inhibitors in a murine model of resistant ovarian cancer.

\section{Methods \\ Animals}

Female C57/Bl6 mice were obtained from Charles Rivers/NCI. Protocols for the use of animals in these studies were reviewed and approved by the Institutional Animal Care and Use Committee of the Lankenau Institute for Medical Research in accordance with the current US Department of Agriculture, Department of Health and Human Service regulations and standards.

\section{Cell Culture}

Luciferase-expressing ID8 (ID8-luc) mouse ovarian carcinoma cells were kindly provided by Dr. Janet Sawicki of the Lankenau Institute of Medical Research. Cell line authentication and IMPACT tests were performed on these cells before use in 
animal experiments. ID8-luc cells were cultured in DMEM supplemented with $4 \%$ fetal bovine serum, $1 \mathrm{x}$ insulin/transferrin/ sodium selenite media supplement (Corning) and 1x Penicillin/ Streptomycin (Cellgro). The cells were freshly thawed from early passage cells, cultured for no more than 2 months, and regularly checked by virtue of their morphologic features to avoid cross-contamination or misuse.

\section{Murine ID8 Tumor Model}

Female C57/Bl6 mice were intraperitoneally (i.p.) injected with $2.0 \times 10^{6}$ ID8-luc cells. To monitor tumor growth and spread throughout the peritoneal cavity, mice were imaged for bioluminescence using an IVIS bioluminescence imager. Three weeks after ID8-luc cells were injected, anti-CTLA-4 therapy was started with $100 \mu \mathrm{g}$ of anti-CTLA-4 antibody (clone 9D9, BioXCell) injected i.p. every third day for a total of three injections. Four weeks after ID8-luc cells were injected, treatment with anti-PD-1 antibody (clone RMP1-14, BioXCell, $200 \mu \mathrm{g}$, i.p.) was initiated with injections every other day for a total of five injections. All mice not receiving anti-CTLA-4 or anti-PD-1 antibodies received corresponding doses of isotype control antibody (BioXCell). When tumor bioluminescence values were approximately $5.0 \times 10^{5}$ photons $/ \mathrm{sec} / \mathrm{cm}^{2}$, treatments with cisplatin $(1 \mathrm{mg} / \mathrm{kg}$, i.p. once weekly) and dabigatran were initiated. Mice were dosed with dabigatran etexilate by oral gavage twice daily $(80 \mathrm{mg} / \mathrm{kg}$ ) Monday through Friday and placed on dabigatran etexilate chow (10 mg/g chow) over the weekends. For survival studies, mice were sacrificed after significant accumulations of ascites were observed in the mice or mice developed adverse effects such as slowed movement or an unkempt, disheveled appearance. Upon sacrifice, ascites fluid was collected, and a final bioluminescence value obtained by opening the peritoneal cavity and imaging it. A section of the peritoneal membrane with ID8 tumors was collected for analysis by flow cytometry. Collected ascites were measured and centrifuged at $300 \mathrm{x}$ g for 5 minutes to precipitate cells. The supernatant was frozen at $-80^{\circ} \mathrm{C}$ for cytokine analyses, and the cell pellets were analyzed by flow cytometry.

\section{Flow cytometry analysis}

Ascites cell pellets were incubated with $5 \mathrm{ml}$ of red cell lysis buffer (0.17 M Tris-HCL, 0.16 M NH4Cl) for $3 \mathrm{~min}$. Cells were spun down and resuspended in FACS buffer (1.5\% heat-inactivated FBS in PBS). Peritoneal tumor samples were minced with scissors and then incubated with collagenase/liberase for 20 min. Samples were spun down and resuspended in $5 \mathrm{ml}$ of red cell lysis buffer for $3 \mathrm{~min}$ and then spun down and resuspended in FACS buffer. Equal numbers of viable cells were stained with the indicated antibodies (F4/80-PE-Cy7 (25-4801-82), CD8-PECy7 (25-0081-82), CD4-PE (12-0081-82), IFN- $\gamma$ (17-7311-82), CD45-PE-Cy5 (15-0451-83) all from eBiosciences and CD206FITC (141704), Biolegend) for $1 \mathrm{~h}$ at $4^{\circ}$ in the dark. Flow-cytometric data were acquired on a BD FACSCanto II and analyzed using FACSDiva software (BD Biosciences). Viable cells were gated based on Fixable Viability Dye eflour 780 staining (Invitrogen).

\section{Intracellular IFN- $\gamma$ staining}

Ascites cell pellets were incubated with $5 \mathrm{ml}$ of red cell lysis buffer for $3 \mathrm{~min}$ and then equal numbers of cells $\left(2.0 \times 10^{6}\right)$ were resuspended in Iscove's Modified Dulbecco's culture media (Gibco) supplemented with $10 \%$ heat inactivated fetal calf serum, $1 \%$ Glutamax (Gibco), 0.5\% gentamycin and $50 \mu \mathrm{M} 2$ - mercaptoethanol. Cells were treated for 4 hours at $37^{\circ} \mathrm{C}$ with ionomycin (500 ng/ml) and phorbol myristate acetate (PMA) (50 ng/ml) to stimulate activated $\mathrm{T}$ cells to produce IFN- $\gamma$ in the presence of Brefeldin A (eBioscience) to block cytokine secretion. Cells were surface stained with PE-Cy7-conjugated anti-CD8 $a$ and PE-Cy5-conjugated anti-CD45 in the presence of Brefeldin A. Cells were fixed, permeabilized, and intracellularly stained with APC-conjugated anti-IFN- $\gamma$ (eBioscience). Flow-cytometric data were acquired on a BD FACSCanto II and analyzed using FACSDiva software (BD Biosciences). Viable cells were gated based on Fixable Viability Dye eflour 780 staining.

\section{Cytokine analyses}

Ascites samples were spun at $300 \mathrm{~g}$ for 10 minutes to pellet cells. Ascites supernatants were collected and analyzed for tumor necrosis factor-alpha (TNF- $\alpha$ ), monocyte chemoattractant protein-1 (MCP-1), IL-6, and IL-10 using the Mouse Inflammation Cytometric Bead Array reagents (BD Biosciences, San Jose, CA) and TGF- $\beta$ using the TGF- $\beta$ flex set (BD Biosciences, San Jose, CA) and flow cytometry as per the manufacturer's protocol.

\section{Statistics}

All in vivo experiments were carried out using multiple animals ( $n=7-10$ per experimental group) in 3 separate experiments. All in vitro experiments were performed in at least triplicate, and data compiled from 2-3 separate experiments. Analyses were done using a 1-way ANOVA with a Tukey test for multiple comparison correction. 
Survival rates were analyzed using the Kaplan-Meier method and evaluated with the log- rank test with Bonferroni correction. For analyzing ID8 tumor spread over time, a linear mixed-effects model and a linear growth model were fit to assess the change in average radiant flux over time among the control and three treatment groups. The models contained the main effects of treatment over time and included the interaction between the main effects. Additionally, they were fit using a random-intercept, which allowed each mouse to have a different baseline radiant flux at day 29. Due to the significant interaction, post-hoc tests were performed to identify significant differences among treatments, and all p-values were adjusted using a Bonferroni correction. All tests were two-sided and the significance level was $\mathrm{p} \leq 0.05$

\section{Results}

\section{Administration of cisplatin and dabigatran decrease tumor burden and increase IFN- $\gamma$ production in CD8+ T-cells during the early stages of tumor progression}

We evaluated the effect of cisplatin or cisplatin/dabigatran etexilate $(\mathrm{C} / \mathrm{D})$ co-treatment with immune checkpoint blockade (ICB) on tumor progression and survival using the ID8 tumor model of ovarian cancer, given that the two therapies modulate the immune response to cancer cells by different, and potentially, complementary mechanisms. Studies conducted using the ID8 model, a highly clinically relevant murine model of ICB-resistant ovarian cancer, have shown that treatment with anti-PD-1 monoclonal antibody (mAb) alone is ineffective in preventing peritoneal tumor growth [29]. At four weeks, daily treatment with $\mathrm{C} / \mathrm{D}$ or vehicle control was initiated along with anti-PD-1 mAb or control isotype mAb. To evaluate tumor burden and the immune response at an early time point during disease progression, mice were sacrificed after 2 weeks of treatment. The tumor growth of the luciferase-expressing ID8 tumor cells was measured by bioluminescence. Whereas treatment with cisplatin, dabigatran, or anti- PD-1 mAb individually had no effect on overall tumor burden, co-treatment with cisplatin and dabigatran with or without anti-PD-1 mAb significantly reduced ID8 tumor burden even after only 2 weeks of treatment (Figure 1A). Interestingly, only mice treated with cisplatin plus dabigatran had significantly higher levels of peritoneal INF- $\gamma$ producing CD ${ }^{+}$T-cells compared to that in mice treated with vehicle control, anti-PD-1 mAb, dabigatran, or cisplatin alone (Figure 1B). Co-treatment of anti-PD-1 mAb with cisplatin did not increase the levels of peritoneal INF- $\gamma$ producing $\mathrm{CD} 8^{+}$T-cells as $\mathrm{did}$ $\mathrm{C} / \mathrm{D}$ co-treatment (Figure $1 \mathrm{~B}$ ). Because cisplatin treatment alone or co-treatment with cisplatin and anti-PD-1 mAb demonstrated a little significant effect on tumor spread and INF- $\gamma$ producing CD8 ${ }^{+}$T-cells after both 2 and 4 weeks of treatment (Figure 1 and data not shown), C/D co-treatment was used in additional tumor experiments.

\section{Co-treatment with dabigatran, cisplatin, and immune checkpoint inhibitors inhibit ID8 tumor growth and as- cites development in vivo}

To examine what effect co-treatment with $\mathrm{C} / \mathrm{D}$ and ICB had on ID8 tumor progression as tumor burden increased, mice were injected with ID8-luc tumor cells and treated with C/D or C/D plus either anti-CTLA-4 or anti-PD-1 mAb and then sacrificed 10 weeks following tumor injection to allow for direct comparisons between treatment groups. Mono-therapy or cisplatin/anti-PD-1 co-treatment was not included due to a lack of effectiveness (data not shown, Figure 1A, B). Treatment with C/D either with or without anti-CTLA-4 or anti-PD-1 mAb significantly inhibited the progression of ID8-luc tumors (Figure $2 \mathrm{~A}, \mathrm{D})$ compared to vehicle-treated control mice, with the slowest tumor progression in mice administered both $\mathrm{C} / \mathrm{D}$ and ICB. The bioluminescence values for the control mice decreased at the final time point due to quenching of bioluminescence by the large volume of ascites in the control mice compared to mice in the treatment groups (Figure 2A, B). After 10 weeks of treatment, all treatment groups had a significantly lower tumor burden compared to control mice with final peritoneal bioluminescence measurements that were 18, 28, and 33-fold lower than control mice for the C/D only, C/D and anti-CTLA-4 $\mathrm{mAb}$ co-treatment, and C/D and anti-PD-1 mAb co-treatment groups, respectively (Figure $2 \mathrm{C}$ ). A closer look at the final peritoneal bioluminescence of mice receiving $\mathrm{C} / \mathrm{D}$ showed that the addition of anti-CTLA-4 mAb or anti-PD-1 mAb to the treatment schedule further reduced the final peritoneal tumor bioluminescence by $40 \%$ and $50 \%$, respectively, compared to treatment with $\mathrm{C} / \mathrm{D}$ alone (Figure $2 \mathrm{C}$, inset).

A common feature of ovarian cancers is the development of ascites, fluid accumulation in the peritoneal cavity containing a dynamic mixture of growth factors, immune cells and tumor cells [30]. Whereas treatment with C/D significantly reduced ascites accumulation by 3 -fold, co-treatment with anti-CTLA-4 or anti-PD-1 mAb resulted in 5 and 10 -fold reductions in ascites volume, respectively (Figure $2 \mathrm{~B}$ ). 
A

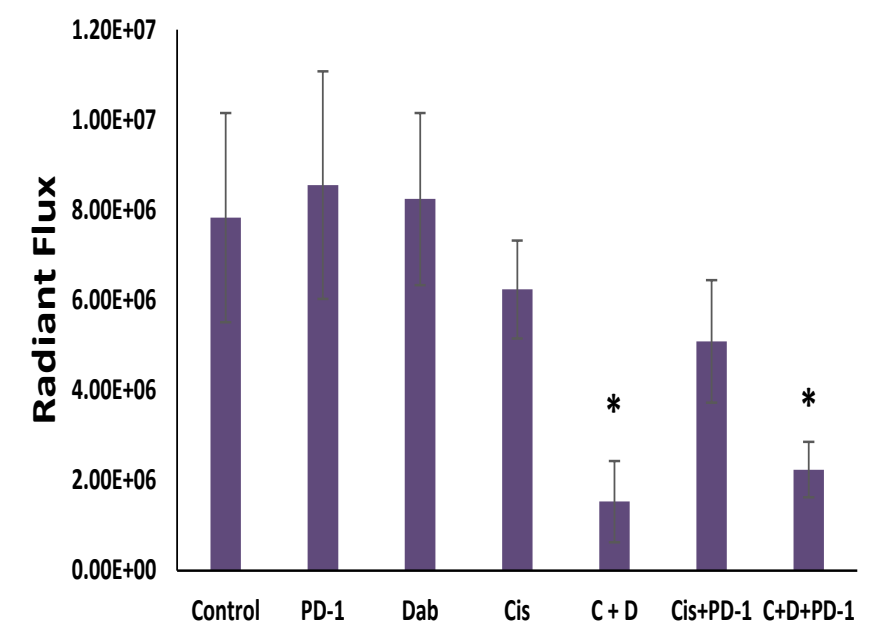

B

$\mathrm{CD}^{+} / \mathrm{IFN}-\mathrm{\gamma}^{+}$

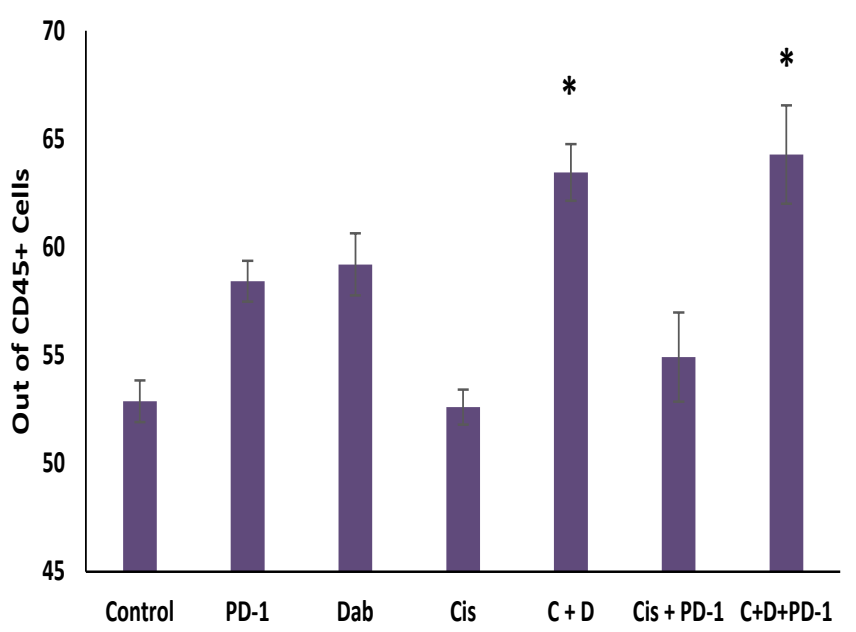

Figure 1: Co-treatment with cisplatin and dabigatran etexilate reduces tumor burden early during tumor progression. Four weeks after mice were i.p. injected with $2.0 \times 10^{6}$ ID8-luc cells, all treatments were initiated. Anti-PD-1 antibodies ( $200 \mu \mathrm{g}$ every two days, five doses total) were i.p. injected. Mice were injected i.p. with cisplatin $(1 \mathrm{mg} / \mathrm{kg})$ once weekly. Dabigatran etexilate was administered by oral gavage twice daily ( $80 \mathrm{mg} /$ $\mathrm{kg}$ ) Monday through Friday, and mice were placed on chow supplemented with dabigatran etexilate (10 mg/g chow) over the weekends. (A) Final tumor loads of mice assessed by bioluminescence imaging of the opened peritoneal cavity six weeks after tumor injection ( 2 weeks of treatment with cisplatin and dabigatran). (B) Cells $\left(2 \times 10^{6}\right)$ isolated from peritoneal lavages were treated for $4 \mathrm{~h}$ at $37^{\circ} \mathrm{C}$ with ionomycin $(500 \mathrm{ng} / \mathrm{ml}) \mathrm{and} \mathrm{PMA}$ $(50 \mathrm{ng} / \mathrm{ml}$ ) to stimulate activated T cells to produce IFN- $\gamma$ in the presence of Brefeldin A to block cytokine secretion. Ascites cells were stained with anti- CD8 $\alpha$ and anti-CD45 antibodies in the presence of Brefeldin A. Cells were then fixed, permeabilized, and intracellularly stained with an anti-IFN- $\gamma$ antibody and analyzed by flow cytometry. $\mathrm{n}=5$ mice per group $\pm \mathrm{SEM} ;{ }^{*}=\mathrm{p}<0.05$ and $\#=\mathrm{p}<0.01$ compared to control vehicle-treated tumor-bearing mice.

Co-treatment with cisplatin and dabigatran plus anti-CTLA-4 or anti-PD-1 antibodies shifts tumor-infiltrating leukocytes to a more anti-tumorigenic profile

The cellular component of the ascites in ID8-luc tumor-bearing mice was analyzed for infiltrating leukocytes by flow cytometry. When mice were sacrificed 10 weeks following tumor injection, there was no difference in the total $\mathrm{CD} 45^{+}$immune cells in the ascites between treatment groups. However, differences were observed in specific subpopulations of ascites leukocytes. In mice receiving $\mathrm{C} / \mathrm{D}$ plus a checkpoint inhibitor, $\mathrm{F} 4 / 80^{+}$macrophages were significantly elevated compared to control mice or mice treated with $\mathrm{C} / \mathrm{D}$ only (Figure $3 \mathrm{~A}$ ). Interestingly, when looking specifically at the pro-tumorigenic $\mathrm{CD} 206^{+} \mathrm{F} 4 / 80^{+} \mathrm{M} 2$-like macrophages, all treatment groups saw significant reductions in M2 macrophages compared to vehicle-treated control mice (Figure 3B). If CD206 ${ }^{+} \mathrm{F} 4 / 80^{+} \mathrm{M} 2$-like macrophages are subtracted from the total $\mathrm{F} 4 / 80^{+}$macrophages, M1 pro-inflammatory macrophages can be identified. The increased ratio of pro-inflammatory M1 macrophages to pro-tumorigenic $\mathrm{CD} 206^{+} \mathrm{F} 4 / 80^{+} \mathrm{M} 2$-like macrophages indicates that the addition of either anti-CTLA-4 or anti-PD-1 mAb to C/D treatment dramatically shifts macrophages to a more pro-inflammatory, potentially anti-tumorigenic phenotype compared to control or C/D-treated mice (Figure 3C).

To further study the cellular compartment of the ascites, we analyzed the ability of $\mathrm{CD}^{+}{ }^{+} \mathrm{T}$-cells in the ascites to produce IFN- $\gamma$, an immune stimulatory response of cytotoxic T-cells to specific antigens. Following stimulation with ionomycin and PMA, IFN- $\gamma$ production by activated $\mathrm{CD} 8^{+}$T-cells was evaluated by flow cytometry. Whereas the overall number of $\mathrm{CD}^{+}{ }^{+} \mathrm{T}$-cells in the ascites of ID8-luc tumor-bearing mice did not change significantly with treatment (data not shown), ex vivo stimulation revealed that $\mathrm{C} / \mathrm{D}$ treatment doubled the percentage of IFN- $\gamma$-producing $\mathrm{CD} 8^{+}$T-cells compared to that in control-treated mice (Figure 3D). The addition of either anti-CTLA-4 or anti-PD-1 therapy to C/D treatment further increased the percentage of IFN- $\gamma$-producing $\mathrm{CD}^{+} \mathrm{T}$ - cells, indicating greater activation of $\mathrm{CD}^{+}{ }^{+} \mathrm{T}$-cells in the ascites of mice treated with $\mathrm{C} / \mathrm{D}$ and ICB (Figure 3D).

To further investigate the effect of ICB on the T-cell response to ID8 ovarian cancer, we isolated tumor-infiltrating leukocytes from tumors on the peritoneal membrane and analyzed them by flow cytometry. Treatment with $\mathrm{C} / \mathrm{D}$ alone significantly increased the number of infiltrating $\mathrm{CD} 8^{+} \mathrm{T}$-cells compared to 


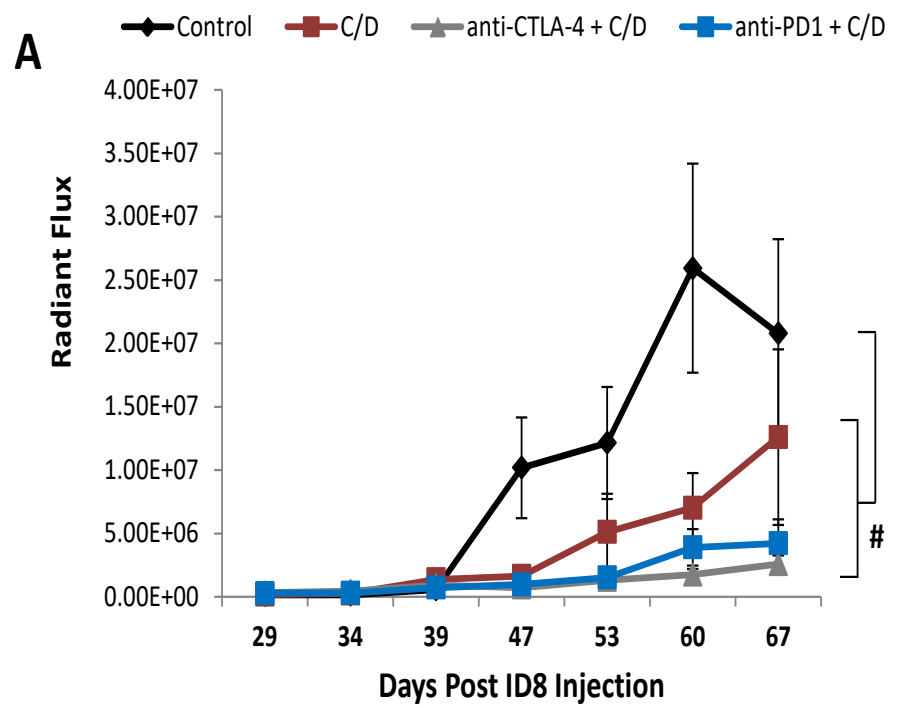

\section{B}

C

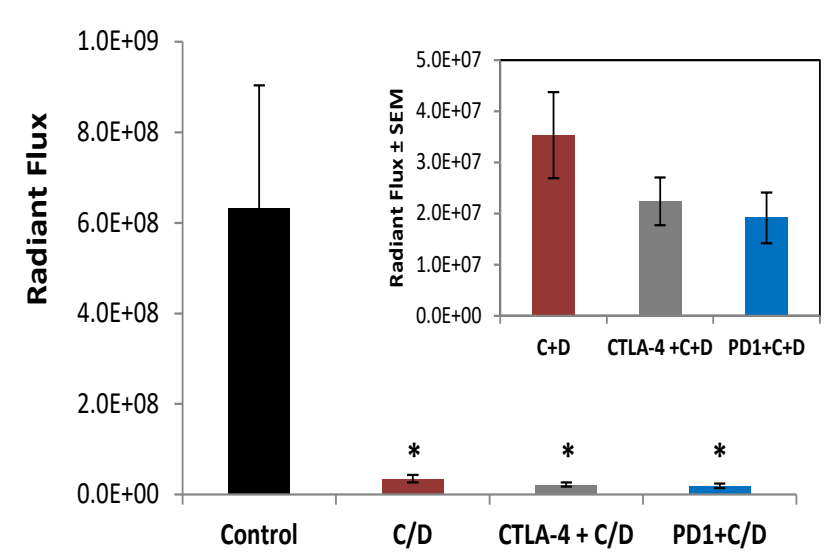

D

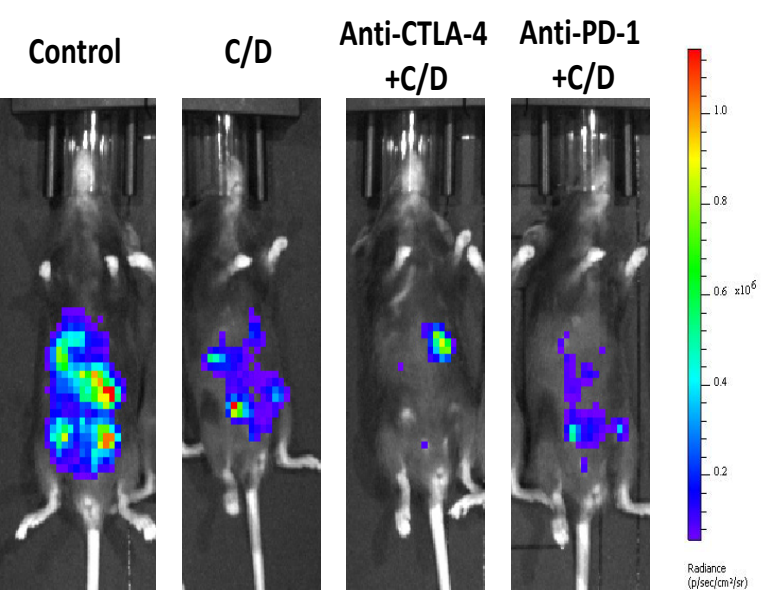

Figure 2: Inhibitory effects of cisplatin and dabigatran etexilate co-treatment plus either anti-CTLA-4 or anti-PD-1 therapy on ID8 tumor growth and malignant ascites accumulation. Mice were treated as described in Figure 1, apart from anti- CTLA-4 treated mice. Mice in the CTLA-4 groups received three doses of anti-CTLA-4 antibodies starting at week three after ID8 cell injection every third day for three doses. All mice were sacrificed after six weeks of treatment (10 weeks after tumor cellinjection).(A) Representative quantification of ID8-luc tumor burden by bioluminescence imaging in living mice. (B) Ascites volume was determined upon sacrifice. (C) The final tumor loads were assessed by bioluminescence imaging of the opened peritoneal cavity. Insert is an enlarged figure of bioluminescence for mice treated with C/D \pm immune checkpoint inhibitors. (D) Representative quantification of ID8-luc tumor burden by bioluminescence imaging in living mice. Mice were sacrificed at 10 weeks post tumor injection. $\mathrm{n}=7-10$ mice per group $\pm \mathrm{SEM} ;{ }^{*}=\mathrm{p}<0.05$ and $\#=\mathrm{p}<0.01$ compared to control vehicle-treated tumor-bearing mice.

that in control mice (Figure 3E). Co-treatment of C/D with anti-CTLA-4 or anti-PD-1 mAb further increased the number of tumor-infiltrating $\mathrm{CD}^{+}{ }^{+}$T-cells. Conversely, co-treatment with immune checkpoint inhibitors in addition to C/D dramatically reduced levels of $\mathrm{CD} 4^{+} \mathrm{T}$-cells in both ascites (Supplemental Figure S1) and tumors (Figure 3F) suggesting that while immune checkpoint inhibition increases the number of CD ${ }^{+}$T-cells and the percentage of those $\mathrm{CD}^{+}{ }^{+}$effector T-cells producing IFN- $\gamma$, the number of suppressive $\mathrm{CD} 4^{+} \mathrm{T}$-cells, such as Tregs, may also be decreased.
Co-treatment with cisplatin and dabigatran and anti-CTLA-4 or anti-PD-1 antibody decreases cytokines in the ascites

Tumors develop many mechanisms to evade immune responses including the secretion of inhibitory cytokines such as tumor growth factor $\beta$ (TGF- $\beta$ ) and IL-10 in addition to inhibitor cell types such as M2 macrophages or Tregs [31]. Cell-free ascites from ID8 tumor-bearing mice was analyzed by cytokine bead arrays using flow cytometry. High levels of the cytokines, TNF- $\alpha$, MCP-1, IL- 6 and TGF- $\beta$, were observed in the ascites of untreated ID8 tumor-bearing mice (Figure 4). Treatment with C/D significantly reduced levels of TNF- $\alpha$, MCP-1, IL-10, and TGF- $\beta$ but not IL-6 (Figure 5). Co-treatment with C/D plus ei- 
ther anti-CTLA-4 or anti-PD-1 mAb further lowered levels of TNF- $\alpha$ and MCP-1 compared to that in mice treated with C/D alone. However, only co-treatment with anti-PD-1 and C/D significantly reduced levels of IL- 6 in the ascites compared to control mice.

Co-treatment with dabigatran etexilate, cisplatin, and an immune checkpoint inhibitor confers a durable survival advantage over immune checkpoint blockade alone

To determine if the changes observed in mice receiving co-treatment with $\mathrm{C} / \mathrm{D}$ and ICB would confer a survival advantage over $\mathrm{C} / \mathrm{D}$ or ICB alone, mice were treated as indicated in Figure 5A. Mice in the vehicle control group began to develop ascites by week 9, surviving only until week 13 (Figure 5B). Treatment with only anti-CTLA- 4 or anti-PD-1 mAb demonstrated no survival effect, indicating that the ID8-luc tumors are resistant to immune checkpoint inhibition alone. Treatment with $\mathrm{C} / \mathrm{D}$ alone significantly enhanced the survival of the mice until week 18, more than a month after mice in the control group or mice receiving ICB alone. However, the most dramatic survival effects were seen in mice receiving both ICB and C/D treatments. At week 18,1/8 mice in the C/D plus anti-CTLA-4 group and 2/7 mice in the $\mathrm{C} / \mathrm{D}$ plus anti-PD-1 treatment group were still alive with low tumor bioluminescence. To determine how durable the response to treatment was, treatment with $\mathrm{C} / \mathrm{D}$ was discontinued in these surviving mice. Tumor bioluminescence continued to be stable at very low levels for an additional 1-2 months in these surviving mice, with mice co-treated with $\mathrm{C} / \mathrm{D}$ and anti-PD-1 $\mathrm{mAb}$ surviving for up to three months following discontinuation of all treatment (Figure 5B). Overall, these results show that C/D co-treatment with ICB enhanced survival and anti-tumor efficacy that was also accompanied by decreases in immunosuppressive M2-macrophages, decreases in pro-tumorigenic cytokines, and corresponding increases in $\mathrm{CD}^{+} \mathrm{T}$-cell infiltration of ID8 tumors and increased IFN- $\gamma$ production.

\section{Discussion}

Previously, we showed significantly greater anti-tumor efficacy with dabigatran etexilate and cisplatin co-treatment that was accompanied by a decrease in immunosuppressive myeloid cell populations and pro-tumorigenic cytokines as well as a concomitant increase in $\mathrm{CD}^{+}$effector T-cell activity in the tumor ascites [27]. Here we show that co-treatment with chemotherapeutic cisplatin and the thrombin inhibitor dabigatran significantly enhances the efficacy of ICB in a murine model of ovarian cancer that is resistant to ICB alone. Of particular significance was that approximately a third of the mice treated with $\mathrm{C} / \mathrm{D}$ plus
anti-PD-1 mAb survived, without treatment, for an additional three months beyond the survival of mice treated with $\mathrm{C} / \mathrm{D}$ alone.

Thrombin has the potential to directly modulate the immune response to the developing tumor. The chronic pro-inflammatory state in the tumor microenvironment has been shown to induce thrombin expression $[32,33]$ mediated by the pro-tumorigenic, pro-inflammatory cytokine IL-6 [34]. Thrombin signals through the PAR-1 receptor which is abundantly expressed in the tumor microenvironment including infiltrating immune cells [35]. Ablation of PAR-1 from the tumor microenvironment, but not the tumor, dramatically reduces tumor growth and metastasis in multiple tumor models, in part by reducing the infiltration of M2-like macrophages into the tumor [23]. We have previously shown that ID8 tumor cells stimulated with thrombin secrete high levels of MCP-1 and that conditioned media from thrombin-treated ID8 tumor cells promotes the conversion of macrophages into an arginase-expressing M2-like phenotype [27]. In this study, we demonstrated that inhibition of thrombin with dabigatran etexilate in conjunction with administration of cisplatin significantly reduces levels of MCP-1 and M2 macrophages in the ascites of ID8 tumor-bearing mice, and these levels are further reduced following co-treatment with anti-CTLA- 4 or anti-PD-1 mAb.

Gold standard treatment for women with ovarian cancer is surgical debulking followed by platinum-based chemotherapy, such as cisplatin. Cisplatin kills cancer cells by cross-linking DNA and inhibiting mitotic cell division. It was originally believed that chemotherapy negatively impacted anti-tumor immunity in the host, but that dogma has been challenged by recent studies. Grabosch et.al. [36] recently demonstrated that the treatment of epithelial ovarian cancers in vitro and in vivo with cisplatin increases tumor immunogenicity by causing novel mutations in the ovarian cancer cells, increasing antigen presentation, and stimulating the accumulation of cytotoxic $\mathrm{CD} 8^{+} \mathrm{T}$-cells in epithelial ovarian tumors. Anti-PD-1 therapy tends to work best in "hot" tumors that have a high level of T-cell infiltration while increased immunogenicity of the tumor cells may give the immune system a better target. We observed that tumor-infiltrating $\mathrm{CD}^{+}{ }^{+} \mathrm{T}$-cells were increased in C/D-treated mice compared to control mice. It is important to note that only $\mathrm{C} / \mathrm{D}$ co-treatment, but not single treatment with cisplatin, dabigatran or anti-PD-1 antibodies alone, increased IFN- $\gamma$-producing $\mathrm{CD} 8^{+} \mathrm{T}$ cells and decreased myeloid immunosuppressive cells in ID8 tumor-bearing mice. 


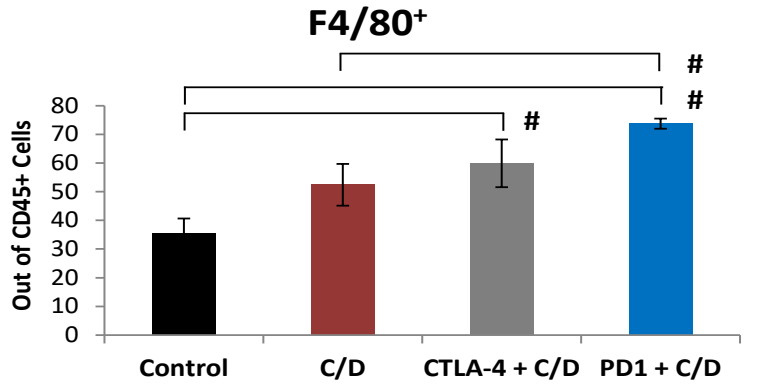

C

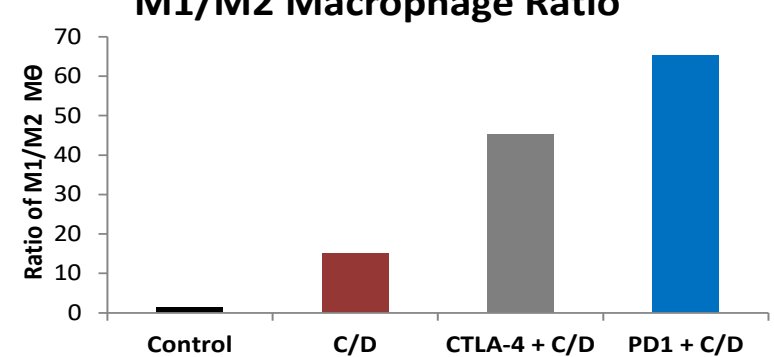

E

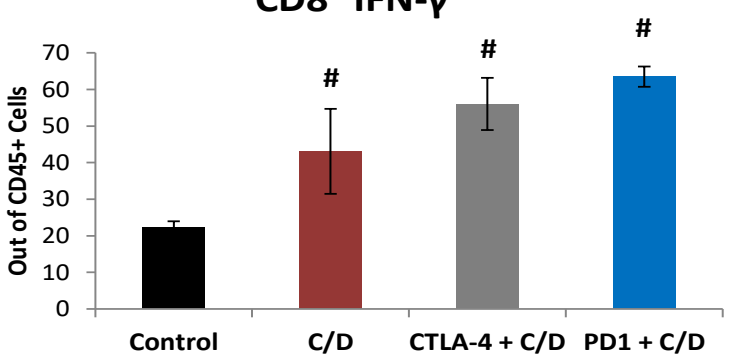

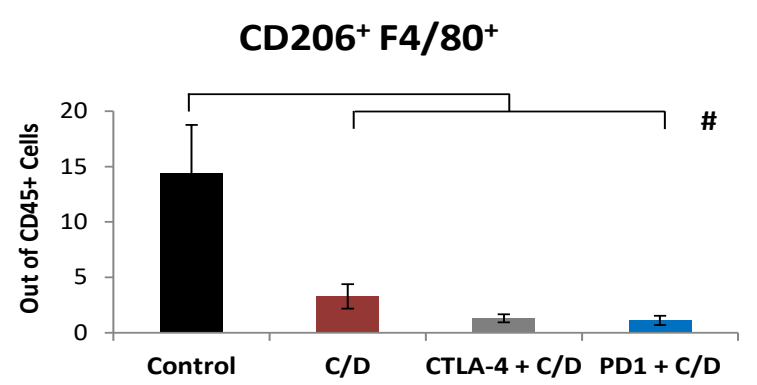

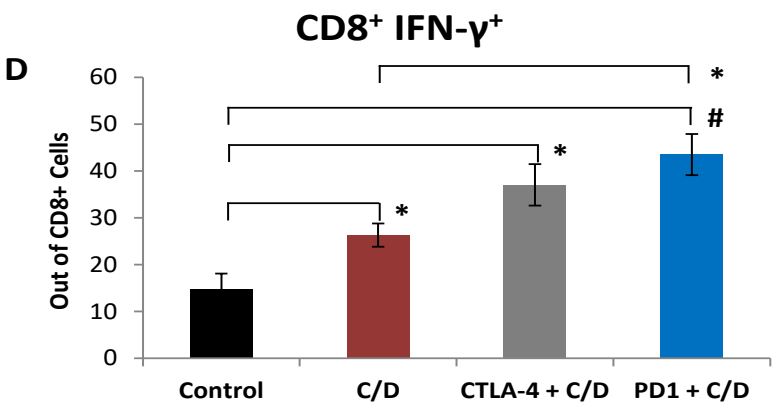

$\mathrm{CD}^{+}$

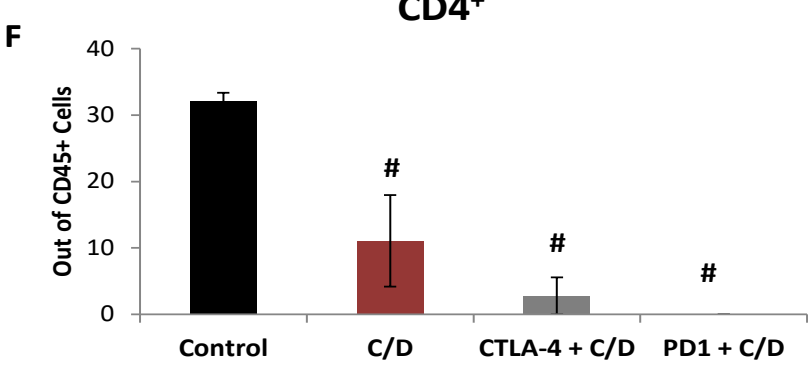

Figure 3. Cisplatin and dabigatran etexilate co-treatment plus either anti-CTLA-4 or anti-PD-1 therapy increases cytotoxic T-cell IFN- $\gamma$ production and decreases M2 macrophage levels in the ascites ID8-luc tumor-bearing mice. Upon sacrifice, ascites was removed from ID8 tumor-bearing mice and spun at $300 \mathrm{~g}$ for 10 minutes to isolate the cellular component. (A-B) CD45 ${ }^{+}$ascites cells were stained and analyzed by flow cytometry for the percentage of the indicated leukocytes. (C) The ratio of pro-inflammatory M1 macrophages to pro-tumorigenic M2 $\mathrm{F} 4 / 80^{+} \mathrm{CD} 206^{+}$macrophages where M1 macrophages are [(total F4/80 ${ }^{+}$macrophages) $-\left(\mathrm{F} 4 / 80^{+} \mathrm{CD} 206^{+} \mathrm{M} 2\right.$ macrophages) . (D) Ascites cells $\left(2 \times 10^{6}\right)$ were treated for $4 \mathrm{~h}$ at $37^{\circ} \mathrm{C}$ with ionomycin $(500 \mathrm{ng} / \mathrm{ml})$ and PMA $(50 \mathrm{ng} / \mathrm{ml})$ to stimulate activated T cells to produce IFN- $\gamma$ in the presence of Brefeldin A to block cytokine secretion. Ascites cells were stained with anti- CD8 $\alpha$ and anti-CD45 antibodies in the presence of Brefeldin A. Cells were then fixed, permeabilized, and intracellularly stained with an anti-IFN- $\gamma$ antibody and analyzed by flow cytometry. (E-F) Upon sacrifice, ID8 tumors on the peritoneal wall of tumor-bearing mice were excised, digested and processed into a single cell suspension for analysis by flow cytometry. $\mathrm{CD} 45^{+}$cells were analyzed for the percentage of the indicated tumor-infiltrating leukocytes. $\mathrm{n}=7-10 \mathrm{mice}$ per group $\pm \mathrm{SEM}$; ${ }^{\star}=\mathrm{p}<0.05$ and $\#=\mathrm{p}<0.01$ compared to control vehicle-treated tumor-bearing mice or indicated groups.

Blockade of CTLA-4 is thought to primarily impact $\mathrm{T}$-cell activation in the tumor microenvironment where CTLA-4 expressing Tregs accumulate and can remove CD80 and CD86 from the surface of antigen-presenting cells [37]. We observed that $\mathrm{C} / \mathrm{D}$ treatment dramatically reduced $\mathrm{CD} 4^{+} \mathrm{T}$-cells in both the ascites and tumors of ID8 tumor-bearing mice, potentially blunting the efficacy of anti-CTLA-4 immune checkpoint blockade. On the other hand, several aspects of PD-1 biology make PD-1 blockade an effective co-treatment with C/D. PD-1 expressing, tumor-infiltrating T-cells are inhibited by PD-L1 expressing tumor cells and other infiltrating leukocytes suggesting that anti- PD-1 therapy primarily boost the effector stage of the immune response [38]. C/D co-treatment increased intra-tumoral levels of $\mathrm{CD}^{+}$T-cells generating a "hotter" tumor for anti-PD-1 mAb therapy to stimulate. Although $\mathrm{CD}^{+}$T-cells can produce elevated levels of IFN- $\gamma$ leading to up regulation of PD-L1 in tumor cells and PD-1 mediated suppression of CD8 ${ }^{+}$ T-cell cytotoxicity, $\mathrm{PD}-1$ blockade can reverse this possible effect of $\mathrm{C} / \mathrm{D}$ co-treatment to enhance anti-tumor efficacy.

The present findings demonstrate that $\mathrm{C} / \mathrm{D}$ co-treatment significantly enhanced the anti-tumor efficacy of ICB in a murine model of ICB-resistant ovarian cancer by modulating multiple aspects of the immune response to tumor growth. 
TNF- $\alpha$

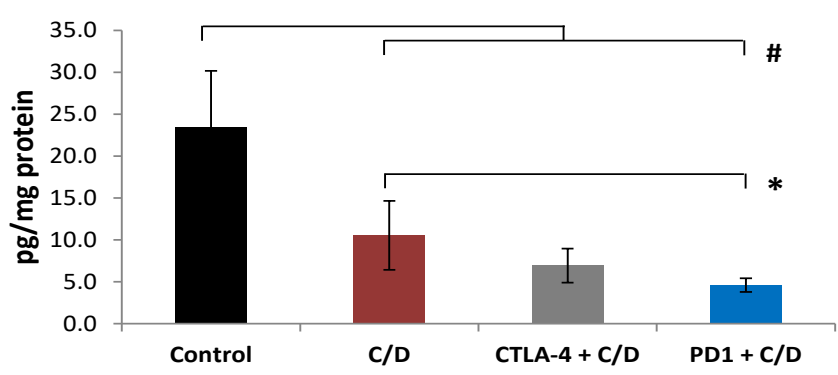

IL-10

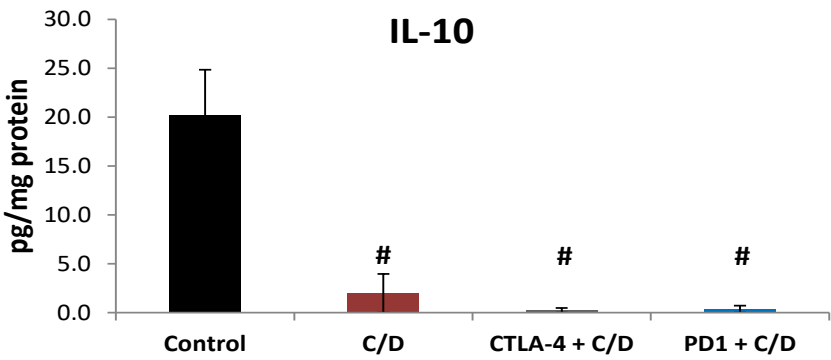

TGF- $\boldsymbol{\beta}$

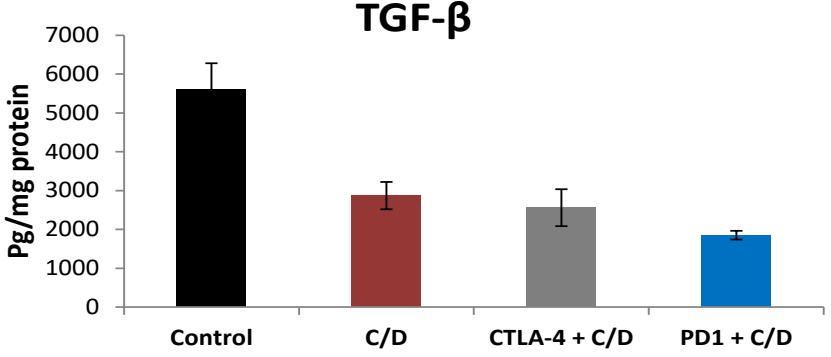

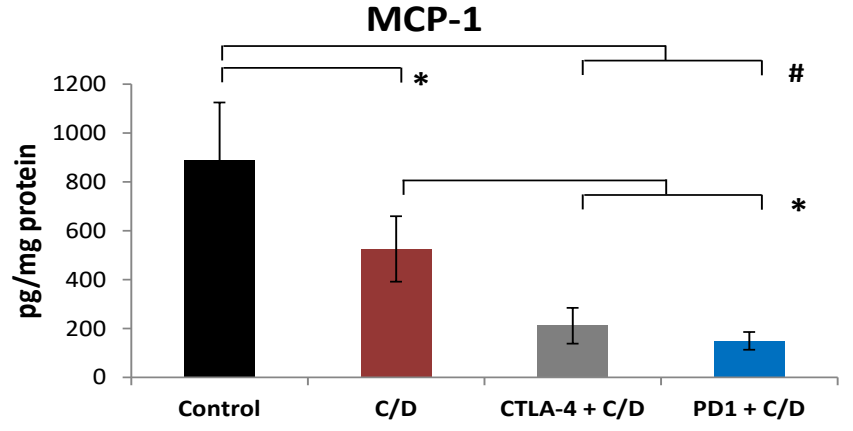

IL-6

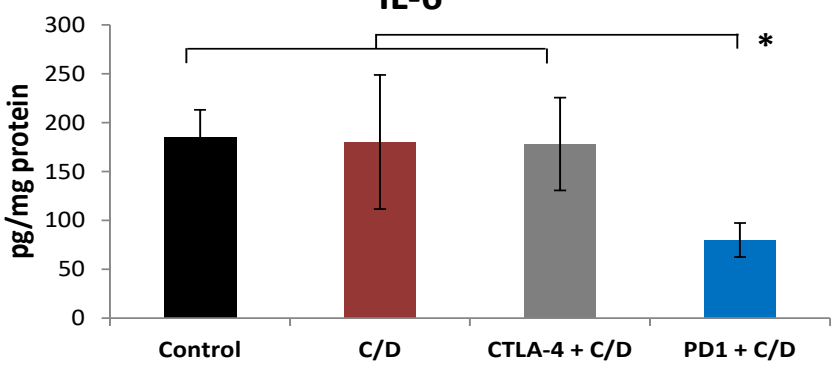

Figure 4. Cisplatin and dabigatran etexilate co-treatment plus either anti-CTLA-4 or anti-PD-1 therapy reduce levels of pro-tumorigenic cytokines in the ascites. Upon sacrifice, ascites were removed and spun at $300 \mathrm{~g}$ for 10 minutes to isolate the cell-free component of the ascites which was assayed for levels of TNF- $\alpha$, MCP-1, IL-10, IL- 6 , and TGF- $\beta$ by Cytokine Bead Array. $n=7-10$ mice per group \pm SEM; ${ }^{*}=p<0.05$ and \# $=\mathrm{p}<0.01$ compared to control vehicle-treated tumor-bearing mice or indicated groups.

\section{A}

ID8 Cells

injected

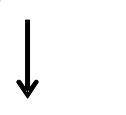

ID8 Treatment Schematic

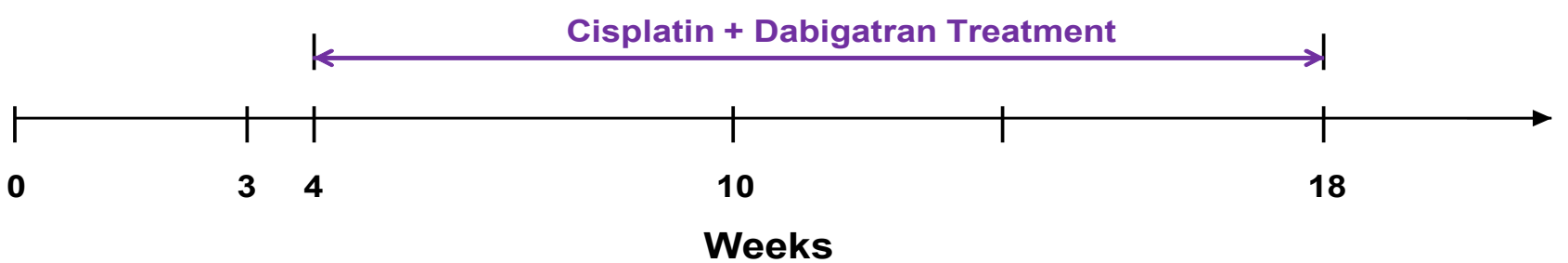

Figure 5: Co-treatment with cisplatin and dabigatran etexilate plus either anti-CTLA-4 or anti-PD-1 therapy confers a survival benefit in mice with ID8 ovarian tumors. (5A) Overall schematic of ID8 ovarian tumor survival experiments. Three weeks after mice were i.p. injected with $2.0 \times 10^{6}$ ID8-luc cells, administration of anti- CTLA-4 antibodies (100 $\mu$ g every three days, three doses total) was initiated. At week4, all other treatments were initiated. Anti-PD-1 antibodies (200 $\mu$ g every two days, five doses total) were i.p. injected. Mice were injected i.p. with cisplatin $(1 \mathrm{mg} / \mathrm{kg}$ ) once weekly. Dabigatran etexilate was administered by oral gavage twice daily ( $80 \mathrm{mg} / \mathrm{kg}$ ) Monday through Friday, and mice were placed on chow supplemented with dabigatran etexilate (10 mg/g chow) over the weekends. Treatment with cisplatin and dabigatran etexilate $(\mathrm{C} / \mathrm{D})$ was terminated at week 18. 


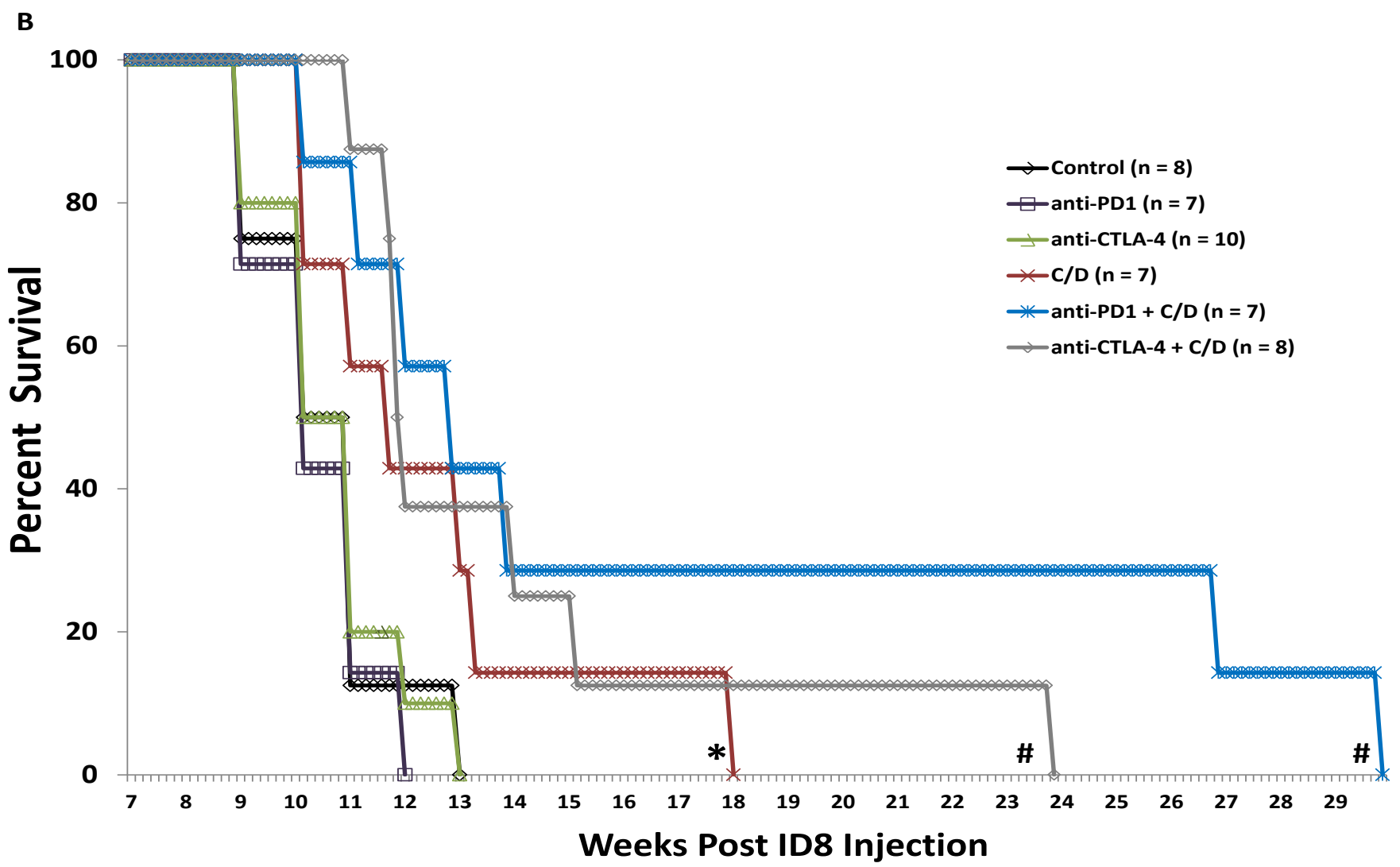

Figure 5B: Kaplan-Meier survival curves of ID8 tumor-bearing mice treated with vehicle, anti-CTLA-4 alone, anti-PD- 1 alone, C/D with or without anti-CTLA-4, or C/D with or without anti-PD-1. $\mathrm{n}=7-10$ mice per group; ${ }^{*}=\mathrm{p}<0.05$ and $\#=\mathrm{p}<0.01$ compared to control vehicle-treated tumor-bearing mice.

Only the combination of cisplatin treatment with the thrombin inhibitor dabigatran significantly increased $\mathrm{CD} 8^{+}$T-cell tumor infiltration while reducing the immunosuppressive microenvironment by decreasing levels of myeloid immunosuppressor cells and multiple immunosuppressive cytokines. The development of new oral anticoagulants such as the thrombin inhibitor dabigatran offers several advantages over vitamin $\mathrm{K}$ antagonists and heparins in that it requires no monitoring has less bleeding risk, and a direct antidote is available [39]. Our findings provide proof-of-concept evidence that the addition C/D with ICB may be a potential new therapeutic treatment combination that further harnesses the immune system for the treatment of advanced ovarian cancer.

\section{Conflicts of Interest and Financial Disclosure}

Susan Gilmour received funding support from Boehringer Ingelheim International GmbH. Joanne van Ryn is employed by but not a shareholder of Boehringer Ingelheim International $\mathrm{GmbH}$. The other authors claim no conflict of interest. 

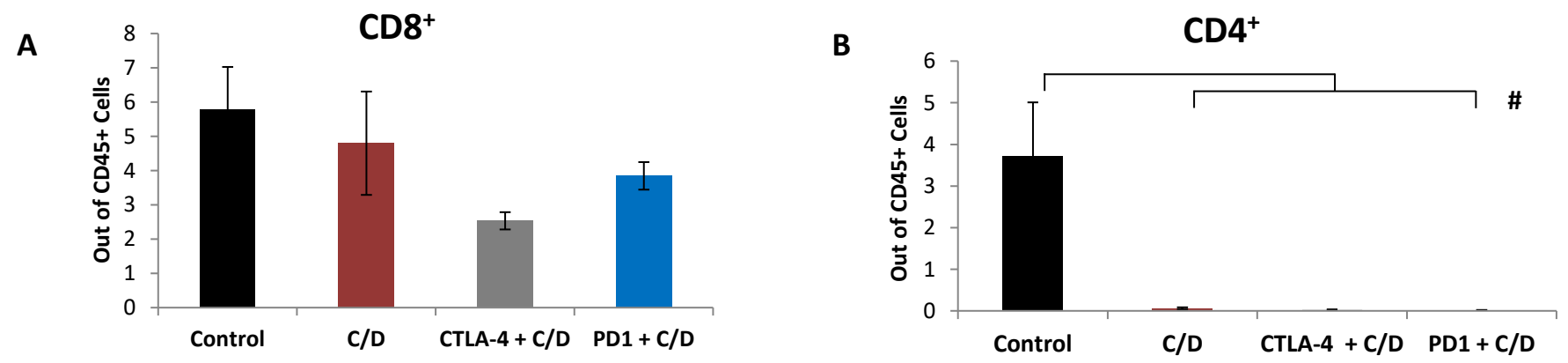

Supplementary Figure 1. Upon sacrifice, ascites was removed from ID8 tumor-bearing mice, and the cellular component was isolated. (A-B) CD45+ ascites cells were gated and analyzed by flow cytometry for the percentage of the indicated CD8+ or CD4+ T-cells. $\mathrm{n}=7-10$ mice per group $\pm \mathrm{SEM} ;{ }^{*}=\mathrm{p}<0.05$ and $\#=\mathrm{p}<0.01$ compared to control vehicle-treated tumor-bearing mice or indicated treatment groups.

\section{References}

1. Siegel RL, Miller KD, and Jemal A (2016) Cancer statistics. CA Cancer J Clin 66: 7-30.

2. Hanker LC, Loibl S, Burchardi N, Pfisterer J, et al. (2012) The impact of second to sixth line therapy on survival of relapsed ovarian cancer after primary taxane/platinum-based therapy. Ann Oncol 23: 2605-2612.

3. Zou W, and Chen L (2008) Inhibitory B7-family molecules in the tumor microenvironment. Nature reviews. Immunology 8: 467-477.

4. Keir ME, Butte MJ, Freeman GJ, and Sharpe AH (2008) PD-1 and its ligands in tolerance and immunity. Annual review of immunology 26: 677-704.

5. Rossi S, Toschi L, Castello A, Grizzi F, Mansi L, and Lopci E (2017) Clinical characteristics of patient selection and imaging predictors of outcome in solid tumors treated with checkpoint-inhibitors. European journal of nuclear medicine and molecular imaging 44: 2310-2325.

6. Gaillard SL, Secord AA, and Monk B (2016) The role of immune checkpoint inhibition in the treatment of ovarian cancer. Gynecol Oncol Res Pract 3: 11.

7. Postel-Vinay S, Aspeslagh S, Lanoy E, Robert C, Soria JC, and Marabelle A (2016) Challenges of phase 1 clinical trials evaluating immune checkpoint-targeted antibodies. Ann Oncol 27:214-224.

8. Barbee MS, Ogunniyi A, Horvat TZ, and Dang TO (2015) Current status and future directions of the immune checkpoint inhibitors ipilimumab, pembrolizumab, and nivolumab in oncology. Ann Pharmacother 49: 907-937.

9. Boland JL, Zhou Q, Martin M, Callahan MK, Konner J, O'Cearbhaill RE, Friedman CF, et al. (2019) Early disease progression and treatment discontinuation in patients with advanced ovarian cancer receiving immune checkpoint blockade.
Gynecol Oncol 152: 251-258.

10. Varki A (2007) Trousseau's syndrome: multiple definitions and multiple mechanisms. Blood 110: 1723-1729.

11. Khorana AA (2010) Venous thromboembolism and prognosis in cancer Thromb Res 125: 490-493.

12. Kasthuri RS, Taubman MB, and Mackman N (2009) Role of tissue factor in cancer. J Clin Oncol 27: 4834-4838.

13. Han LY, Landen CN, Jr, Kamat AA, Lopez A, Bender DP, Mueller P, Schmandt R, Gershenson DM, and Sood AK (2006) Preoperative serum tissue factor levels are an independent prognostic factor in patients with ovarian carcinoma. J Clin Oncol 24: 755-761.

14. Satoh T, Oki A, Uno K, Sakurai M, Ochi H, Okada S, Minami R, et al. (2007) High incidence of silent venous thromboembolism before treatment in ovarian cancer. Br J Cancer 97: 1053-1057.

15. Ebina Y, Uchiyama M, Imafuku H, Suzuki K, Miyahara Y, and Yamada H (2018) Risk factors for deep venous thrombosis in women with ovarian cancer. Medicine 97, e11009.

16. Barni S, Labianca R, Agnelli G, Bonizzoni E, Verso M, Mandala M, Brighenti M, Petrelli F (2011) Chemotherapy-associated thromboembolic risk in cancer outpatients and effect of nadroparin thromboprophylaxis: results of a retrospective analysis of the PROTECHT study. J Transl Med 9, 179.

17. Nierodzik ML, and Karpatkin S (2006) Thrombin induce tumor growth, metastasis, and angiogenesis: Evidence for a thrombin-regulated dormant tumor phenotype. Cancer cell 10: 355-362.

18. Falanga A, Panova-Noeva M, and Russo L (2009) Procoagulant mechanisms in tumour cells. Best Pract Res Clin Haematol 22: 49-60.

19. Labelle M, Begum S, and Hynes RO (2011) Direct signaling between platelets and cancer cells induces an epithelial-mesenchymal-like transition and promotes metastasis. Can- 
cer cell 20: 576-590.

20. Ossovskaya VS, and Bunnett NW (2004) Protease-activated receptors: contribution to physiology and disease. Physiol Rev 84: 579-621.

21. Danckwardt S, Hentze MW, and Kulozik AE (2013) Pathologies at the nexus of blood coagulation and inflammation: thrombin in hemostasis, cancer, and beyond. Journal of molecular medicine (Berlin, Germany) 91: 1257-1271.

22. Degen JL, and Palumbo JS (2012) Hemostatic factors, innate immunity, and malignancy. Thromb Res 129 Suppl 1, S15.

23. Queiroz KC, Shi K, Duitman J, Aberson HL, Wilmink JW, et al. (2014) Protease-activated receptor-1 drives pancreatic cancer progression and chemoresistance. Int J Cancer 135: 2294 2304.

24. Adams GN, Rosenfeldt L, Frederick M, Miller W, Waltz D, Kombrinck K, McElhinney KE, (2015) Colon Cancer Growth and Dissemination Relies upon Thrombin, Stromal PAR-1, and Fibrinogen. Cancer Res 75: 4235-4243.

25. Kopp HG, Placke T, and Salih HR (2009) Platelet-derived transforming growth factor-beta down-regulates NKG2D thereby inhibiting natural killer cell antitumor reactivity. Cancer Res 69: 7775-7783.

26. Palumbo JS, Talmage KE, Massari JV, La Jeunesse CM, Flick MJ, Kombrinck KW(2005) Platelets and fibrin (ogen) increase metastatic potential by impeding natural killer cell-mediated elimination of tumor cells. Blood 105: 178-185.

27. Alexander ET, Minton AR, Peters MC, van Ryn J, and Gilmour SK (2016) Thrombin inhibition and cisplatin block tumor progression in ovarian cancer by alleviating the immunosuppressive microenvironment. Oncotarget 7: 85291-85305.

28. Hauel NH, Nar H, Priepke H, Ries U, Stassen JM, and Wienen W (2002) Structure-based design of novel potent nonpeptide thrombin inhibitors. J Med Chem 45: 1757-1766.

29. Guo Z, Wang X, Cheng D, Xia Z, Luan M, and Zhang S (2014) PD-1 blockade and OX40 triggering synergistically protects against tumor growth in a murine model of ovarian cancer. PLoS One 9, e89350.

30. Ahmed N, and Stenvers KL (2013) Getting to know ovarian cancer ascites: opportunities for targeted therapy-based translational research. Front Oncol 3: 256.

31. Vinay DS, Ryan EP, Pawelec G, Talib WH, Stagg J, Elkord E, Lichtor T, Decker WK, Whelan R L, et al. (2015) Immune evasion in cancer: Mechanistic basis and therapeutic strategies. Semin Cancer Biol 35 Suppl, S185-S198.

32. Danckwardt S, Gantzert AS, Macher-Goeppinger S, Probst HC, Gentzel M, et al. (2011) p38 MAPK controls pro- thrombin expression by regulated RNA 3' end processing. Mol Cell 41: 298-310.

33. Levi M, van der Poll T, and Buller HR (2004) Bidirectional relation between inflammation and coagulation. Circulation 109: 2698-2704.

34. Levi M, and van der Poll T (2010) Inflammation and coagulation. Crit Care Med 38: S26-34.

35. Vergnolle N (2000) Proteinase-activated receptors - novel signals for gastrointestinal pathophysiology. Aliment Pharmacol Ther 14: 257-266.

36. Grabosch S, Bulatovic M, Zeng F, Ma T, Zhang L, Ross M, Brozick J, Fang Y, Tseng G, et al. (2019) Cisplatin-induced immune modulation in ovarian cancer mouse models with distinct inflammation profiles. Oncogene 38: 2380-2393.

37. Curran MA, Montalvo W, Yagita H, and Allison JP (2010) PD-1 and CTLA-4 combination blockade expands infiltrating $\mathrm{T}$ cells and reduces regulatory $\mathrm{T}$ and myeloid cells within B16 melanoma tumors. Proceedings of the National Academy of Sciences of the United States of America 107: 4275-4280.

38. Ahmadzadeh M, Johnson LA, Heemskerk B, Wunderlich JR, Dudley ME, White DE, et al (2009) Tumor antigen-specific CD8 T cells infiltrating the tumor express high levels of PD-1 and are functionally impaired. Blood 114: 1537-1544.

39. Pollack CV, Jr. Reilly P A, van Ryn J, Eikelboom JW, Glund S, Bernstein RA, et al. (2017) Idarucizumab for Dabigatran Reversal - Full Cohort Analysis. N Engl J Med 377: 431-441.

\footnotetext{
Submit your manuscript to a JScholar journal and benefit from:

9 Convenient online submission

ब Rigorous peer review

I Immediate publication on acceptance

- Open access: articles freely available online

9 High visibility within the field

ब Better discount for your subsequent articles

Submit your manuscript at http://www.jscholaronline.org/submit-manuscript.php
} 